\title{
$K$-THEORY OF COMMUTATIVE REGULAR RINGS
}

\author{
ANDY R. MAGID
}

Abstract. Pierce's representation of a commutative regular ring as a sheaf of fields is used to compute the $K$-theory of the ring: $K_{1}$ is units (Robert's Theorem) and $K_{2}$ is generated by symbols.

Pierce [4] shows how a commutative regular (in the sense of von Neumann) ring $R$ can be represented as the ring of all global sections of a sheaf $R$ of fields over a zero-dimensional compact space $X$. Because of the zerodimensionality of $X$, data over the stalks of $R$ can be extended to similar global data, and thus much of the theory of $R$ follows immediately from the corresponding theory for fields. This brief note uses this technique to compute the $K$-theory of $R: K_{0}(R)$ is the ring $C(X, Z)$ of continuous functions from $X$ to the integers $Z$ (this is due to Pierce [4, 16.4, p. 67]); $K_{1}(R)$ is the group of units of $R$ (this is due to Roberts [5, p. 425]); and $K_{2}(R)$ is generated by the universal Steinberg symbols. The proofs use only the fact that the $K_{i}$ are functors of finite type and hence apply to other such functors. This gives new proofs of earlier results on the Brauer group [2, 1.10, p. 117].

We begin by recalling Pierce's general construction: to each commutative ring $R$ is associated a compact, zero-dimensional Hausdorff space $X(R)$ and a sheaf $\mathscr{R}$ of connected rings on $X(R)$ such that $R=\Gamma(X(R), \mathscr{R})$ $[4,4.4$, p. 17].

When $R$ is regular, this is just the usual sheaf of local rings on $\operatorname{Spec}(R)$ $[6,2.4]$.

Now suppose $F$ is an abelian group valued functor on the category of commutative rings. Then $F \circ \mathscr{R}$ is a presheaf of abelian groups on $X(R)$. We denote its associated sheaf by $\# F$. Further study of $\# F$ requires the following hypothesis.

Definition (SEE $[1,1.5$, p. 24]). $\quad F$ is of finite type if $F$ commutes with finite products and arbitrary direct limits.

Proposition 1. In the above notation, suppose $F$ is of finite type. Then the group of global sections of \#F is isomorphic to $F(R)$ and for each $x$ in $X(R)$, the stalk $(\# F)_{x}$ is isomorphic to $F\left(R_{x}\right)$.

Received by the editors October 6, 1972.

AMS (MOS) subject classifications (1970). Primary 13D15; Secondary 13A30.

Key words and phrases, $K$-theory, von Neumann regular ring, functor of finite type. 
Proof. For the first part, see [2, 1.9, p. 117]. For the second part, $(\# F)_{x}=F \circ \mathscr{R}_{x}=\operatorname{dir} \lim F(\Gamma(U, \mathscr{R}))=F(\operatorname{dir} \lim \Gamma(U, \mathscr{R})) \quad($ since $F$ is of finite type) and $\operatorname{dir} \lim \Gamma(U, \mathscr{R})=R_{x}$, the direct limit being over open neighborhoods of $x$.

We note the proposition applies to the following functors of finite type: $K_{i}, i=0,1,2, K_{2}$ being in the sense of Milnor [3, p. 40], the Picard group, the multiplicative group $G_{m}$ and the Brauer group. (Regarding the latter, see also $[2,1.10$, p. 117].) We also will consider the universal Steinberg symbol functor $[3,11.1$, p. 93] defined as follows: for any commutative ring $T, U s(T)$ is the multiplicative abelian group with one generator $(a, b)$ for each pair of units $a, b$ of $T$ and relations forcing $(a, b)$ to be bimultiplicative and $(a, b)=1$ if $a+b=1$. Clearly $U s$ is a functor of finite type.

Proposition 2. Let $R$ be a commutative regular ring and $F \rightarrow G$ a natural transformation of functors of finite type from commutative rings to abelian groups which is an isomorphism when the rings are fields. Then $F(R) \rightarrow G(R)$ is also an isomorphism.

Proof. The natural transformation $F \rightarrow G$ induces a sheaf morphism $f: \# F \rightarrow \# G$. For each $x$ in $X(R), R_{x}$ is a field $[4,10.3$, p. 41] and we have a commutative diagram

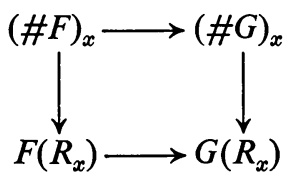

The vertical maps are isomorphisms by Proposition 1 and the lower horizontal map is an isomorphism by hypothesis. Thus the upper map is an isomorphism. Thus $f$, being an isomorphism at each stalk, is an isomorphism and hence induces an isomorphism of global sections. But, by Proposition 1 again, these global sections are $F(R)$ and $G(R)$ respectively.

COROLlaRY 3. Let $R$ be a commutative regular ring. Then:

(a) $K_{0} R=C(X(R), Z)$,

(b) $K_{1} R=G_{m}(R)$,

(c) $K_{2} R=U s(R)$.

Proof. The transformation $K_{0} \rightarrow C(X(), Z)$ is given by the rank homomorphism, the transformation $K_{1} \rightarrow G_{m}$ is given by the determinant and the transformation $U s \rightarrow K_{2}$ is given by symbols [3, p. 74]. These are all isomorphisms for fields, the first two being classical and the third a theorem of Matsumoto $[3,11.1$, p. 93]. Thus Proposition 2 applies and the corollary follows.

Roberts also computes relative $K_{1}$ for commutative regular rings [5, p. 425]. We outline another approach to his result. 
LEMMA 4. Let $R$ be a commutative regular ring, $I$ an ideal of $R$. Then $G_{m}(R) \rightarrow G_{m}(R / I)$ is onto.

Proof. Let $r$ in $R$ go to a unit of $R / I$, and let $R r=R e$ where $e$ is idempotent. $I$ is the intersection of the maximal ideals $M$ containing it, and if $I$ is contained in $M, r$, being a unit modulo $I$, is not, so $e$ is not in $M$ and hence $1-e$ is. This holds for all $M$ containing $I$, so $1-e$ is in $I$. Let $s=1-e+r$. If $s$ is in the maximal ideal $M$, then if $e \in M, r \in M$ so also $1-e \in M$, which is impossible, so $e$ is not in $M$ and $1-e$ is in $M$, hence $r \in M$, hence $e \in M$, again an impossibility. Thus $s$ is in no maximal ideal, hence is a unit congruent to $r$ modulo $I$.

COROllaRY 5. Let $R$ be a commutative regular ring, $I$ an ideal of $R$. Then $K_{1}(R, I)=\operatorname{Kernel}\left(G_{m}(R) \rightarrow G_{m}(R / I)\right)$.

Proof. We have an exact sequence $[3,6.2$, p. 54]

$$
K_{2} R \rightarrow K_{2} R / I \rightarrow K_{1}(R, I) \rightarrow K_{1} R \rightarrow K_{1} R / I
$$

which by Corollary 5 becomes

$$
U s(R) \rightarrow U s(R / I) \rightarrow K_{1}(R, I) \rightarrow G_{m}(R) \rightarrow G_{m}(R / I) .
$$

By Lemma 4 the first map is onto, and the result follows by exactness.

Proposition 1 gives information about rings of continuous functions $C(X, T)$ where $X$ is a compact zero-dimensional topological space and $T$ a commutative ring with the discrete topology. However, a more elementary argument suffices in this special case, which we now record.

Proposition 6. Let $R=C(X, T)$ be as above and let $F$ be a functor of finite type. Then $F(R)$ is isomorphic to $C(X, F(T)$ ) (here $F(T)$ carries the discrete topology).

Proof. A finite partition of $X$ is a cover of $X$ by finitely many disjoint open subsets. The partitions of $X$ are partially ordered by refinement and $X=$ proj $\lim P$ where $P$ ranges over the partitions of $X$. Thus $C(X, T)=$ $\operatorname{dir} \lim C(P, T)$, and $C(P, T)$ is a finite product of copies of $T$. Then since $F$ is of finite type, $F(C(X, T))=\operatorname{dir} \lim F(C(P, T))=\operatorname{dir} \lim C(P, F(T))=$ $C(X, F(T))$.

By the Stone representation theorem, Boolean rings are of the type treated in Proposition 6 (where $T$ is the field of two elements). Thus the $K$-theory of Boolean rings may be computed:

Corollary 7. Let $R$ be a Boolean ring. Then: $K_{0} R=C(X(R), Z)$ and $K_{1}$ and $K_{2}$ of $R$ are trivial.

Proof. The final assertion follows from Steinberg's result [3, 9.9, p. 75] that $K_{2}$ of a finite field is trivial. 
One may also use Proposition 6 to compute $K$-theory of rings of continuous integer valued functions, and also Brauer groups of such rings and Boolean rings as in $[2,1.12$, p. 118].

\section{REFERENCES}

1. M. Artin, Algebraic approximation of structures over complete local rings, Inst. Hautes Études Sci. Publ. Math. 36 (1969), 23-58. MR 42 \#3087.

2. A. Magid, Pierce's representation and separable algebras, Illinois J. Math. 15 (1971), 114-121. MR 42 \#7713.

3. J. Milnor, Introduction to algebraic K-theory, Ann. of Math. Studies, no. 72, Princeton Univ. Press, Princeton, N.J., 1971.

4. R. Pierce, Modules over commutative regular rings, Mem. Amer. Math. Soc. No. 70 (1967). MR 36 \#151.

5. L. Roberts, $K_{1}$ of a commutative von Neumann regular ring, Proc. Amer. Math. Soc. 32 (1972), 425-426.

6. O. Villamayor and D. Zelinsky, Galois theory with infinitely many idempotents, Nagoya Math. J. 35 (1969), 83-98. MR 39 \#5555.

Department of Mathematics, University of Oklahoma, Norman, Oklahoma 73069 